\title{
A Prospective Study of L-Dex Values in Breast Cancer Patients Pretreatment and Through 12 Months Postoperatively
}

\author{
Sheila H. Ridner, PhD, RN, FAAN, Mary S. Dietrich, PhD,,2 Kandace Spotanski, MMHC, \\ Jennifer K. Doersam, MS, Michael S. Cowher, MD, ${ }^{3}$ Bret Taback, MD, Sarah McLaughlin, MD, \\ Nicolas Ajkay, MD, John Boyages, MD, PhD, Louise Koelmeyer, BAppSc(OT), \\ Sarah DeSnyder, $\mathrm{MD}^{8}$, Chirag Shah, $\mathrm{MD}^{9}$, and Frank Vicini, $\mathrm{MD}^{10}$
}

\begin{abstract}
Background: Data regarding pretreatment, bioimpedance spectroscopy (BIS) L-Dex ${ }^{\circledR}$ values for patients newly diagnosed with breast cancer, and longitudinal data 12 months postoperatively are lacking. This study describes L-Dex values at the time of breast cancer diagnosis and maximum L-Dex change within 12 months of surgery. Methods and Results: Patients were enrolled in a parent, clinical trial that compares the effectiveness of BIS for early detection of breast cancer-related lymphedema to tape measurement. A total of 280 women with a pretreatment and at least one postoperative L-Dex measurement (within 12 months of surgery) were included. Pretreatment L-Dex readings were compared with population norms and maximum L-Dex changes within 12 months were examined. An L-Dex U400 device was used to obtain BIS measurements. The documented normative mean value using this device is 0.00 , which is at the 49th percentile for this sample. Approximately $6 \%$ of patients had a pretreatment L-Dex value of $\geq 7.0 ; 1.8 \%$ had an L-Dex value $\geq 10.0$. For 12 months, $17.1 \%(n=48)$ of patients had a maximum change in L-Dex value from pretreatment of $\geq 7.0 \mathrm{~L}$-Dex units, suggestive of clinical lymphedema.

Conclusions: At the time of breast cancer diagnosis, L-Dex values are similar to normative values. Identified maximum changes in L-Dex values 12 months postoperatively suggest that frequent L-Dex measurements during that time frame are of potential clinical benefit. Our findings are consistent with research supporting an L-Dex value of $\geq 7$ as indicative of clinical lymphedema with subclinical lymphedema logically occurring at somewhat lower likely, near $\geq 6.5$.
\end{abstract}

Keywords: breast cancer, lymphedema, bioimpedance, detection

\section{Introduction}

B IOIMPEDANCE SPECTROSCOPY (BIS) emerged as an assessment tool for breast cancer-related lymphedema (BCRL) in the early 1990s yielding data regarding impedance ratios between an at-risk limb and a healthy contralateral limb. ${ }^{1}$ At that time, tape measurement (TM)/circumferential measurement and water displacement were two of the primary measurement methods for arm lymphedema ${ }^{1,2} \mathrm{Al}-$ though convention in medicine is to use a $95 \%$ confidence interval $(\mathrm{CI})$ ( = 1.95 standard deviation [SD] from mean) as a reference range for abnormal findings, ${ }^{3}$ earlier work in BIS

\footnotetext{
${ }^{1}$ School of Nursing, Vanderbilt University, Nashville, Tennessee.

${ }^{2}$ Department of Biostatistics, Vanderbilt Ingram Cancer Center, Vanderbilt University Medical Center, Nashville, Tennessee.

${ }^{3}$ Department of Surgery, Allegheny General Hospital, Pittsburgh, Pennsylvania.

${ }_{5}^{4}$ Division of Breast Surgery, Department of Surgery, Columbia University Medical Center, New York, New York.

${ }^{5}$ Section of Surgical Oncology, Mayo Clinic, Jacksonville, Florida.

${ }^{6}$ Department of Surgical Oncology, University of Louisville, Louisville, Kentucky.

${ }^{7}$ Faculty of Medicine and Health Sciences, Macquarie University, New South Wales, Australia.

${ }^{8}$ Division of Surgery, Department of Breast Surgical Oncology, MD Anderson Cancer Center, Houston, Texas.

${ }^{9}$ Department of Radiation Oncology, Taussig Cancer Institute, Cleveland Clinic, Cleveland, Ohio.

${ }^{10}$ Michigan Healthcare Professionals, 21 st Century Oncology, Farmington Hills, Michigan.
} 
recommended a more conservative normal range for the impedance ratios with regard to lymphedema. This range established 3 SDs from a healthy normal ratio mean as an indicator of lymphedema. ${ }^{1}$ These ratios were later converted to a new metric, the L-Dex ${ }^{\circledR}$ unit with an L-Dex value of +10 corresponding to 3 SDs above healthy population norms; it should be noted that this cutoff is inferential, rather than correlative with other diagnostic techniques.

When BIS was initially developed for use as a lymphedema measurement tool, the construct of subclinical lymphedema was not in the scientific literature, but reports that BIS could predict eventual development of clinical lymphedema began to emerge in the early 2000s. ${ }^{4}$ Based upon the work of Stout et al. in 2008 and subsequent data, the concept of subclinical breast cancer lymphedema was proposed. ${ }^{5}$ Specifically, Stout et al. advocated that a $>3 \%$ volume change postsurgery, in contrast to the $5 \%-10 \%$ change traditionally used to diagnose clinical lymphedema, might be indicative of subclinical lymphedema. In addition, the Stout team purported that early compression sleeve application might prevent clinical lymphedema, applying such a lower threshold as a prevention intervention trigger. BIS was also recommended by the authors as a possible measurement method to evaluate subclinical lymphedema. ${ }^{5}$ Shortly after the completion of the Stout study, BIS was indeed put forth as a desirable measurement method to identify subclinical lymphedema. ${ }^{6}$ This positioning was based primarily on work completed by Ward et al., which found BIS to be more sensitive than TM in detecting lymphedema. ${ }^{7}$

Despite its established value in assessing clinical and subclinical lymphedema, the widespread use of BIS in clinical settings has been somewhat limited. In part, this may be due to concerns from clinicians about the ability of BIS to identify actual cases of lymphedema by using an absolute quantitative value as compared with a change in values over time. In addition, pretreatment measurement to establish BIS baselines is not a common practice in most clinical settings. This creates an environment in which it is virtually impossible to routinely determine change over time.

BIS research is rapidly advancing. Recent publications now advocate that clinical lymphedema is present when there is an L-Dex reading approximating 7, or alternatively, a change $\geq 7$, representative of 2 SDs above the mean established in earlier studies (Table 1). ${ }^{1,4,8-11}$ As an example, in 2013 , Fu et al. reported that the L-Dex unit ratio of $\geq 7.1$ discriminated between at-risk breast cancer survivors and breast cancer survivors with established lymphedema. ${ }^{11}$ The team also reported that the $\geq 7.1 \mathrm{~L}-$ Dex unit threshold missed $20 \%$ of established lymphedema. Ward et al. reported similar findings. ${ }^{8}$ This body of work now aligns the diagnostic standards for clinical lymphedema when using BIS with standards commonly used across medical fields for diagnosis of other medical conditions. ${ }^{3}$ Owing to the recent timing of this change, specific studies have yet to be conducted to establish a clear L-Dex reading/change from baseline that is indicative of subclinical BCRL. However, in keeping with Stout's original premise that subclinical lymphedema occurs at a level below that of clinical lymphedema, these recent findings, coupled with human ethics concerns, support the need to consider the presentation of subclinical lymphedema at an L-Dex reading/change of $\leq 7.1$ if noninvasive, pre- vention interventions advocated by Stout et al. are to be successful. 5

Remaining absent in the current BIS literature are critical data regarding normative L-Dex values for breast cancer patients before treatment and longitudinal patterns of L-Dex change up to 12 months after surgery, the time period when a high percentage of those patients manifest increased swelling. ${ }^{12}$ These gaps leave unanswered questions that potentially compromise patient care and the development of prospective BCRL surveillance programs that utilize BIS to detect subclinical and clinical lymphedema.

In conjunction with an ongoing stratified randomized clinical trial, we have followed the L-Dex readings in a large cohort of patients with breast cancer from presurgery through 12 months postsurgery. The purpose of this analysis was to evaluate pretreatment, baseline L-Dex readings collected prospectively as compared with established population norms, and to evaluate maximum L-Dex changes from each patient's baseline L-Dex value in those with at least one L-Dex measurement within the first 12 months postsurgery.

\section{Materials and Methods}

Patients were enrolled in a two-group, parent, randomized clinical trial that aims to evaluate the effectiveness of BIS for early detection and prevention of clinical lymphedema as compared with TM. Patients were recruited from local breast centers in Australia and the United States. Initial inclusion criteria consisted of age $\geq 18$ with histologically confirmed stage I-III breast cancer or ductal carcinoma in situ with planned surgery. Preliminary exclusion criteria included a history of any type of breast cancer treatment or lymphedema and planned bilateral surgery. The study was conducted in compliance with the ethical standards of the Helsinki Declaration. Institutional Review Board (IRB) and Scientific Review Committee (SRC) approvals were both obtained from Vanderbilt University (coordinating center) and Vanderbilt Ingram Cancer Center before commencement of the parent study. All sites also obtained local IRB and SRC approvals as required. All patients provided informed consent before data collection. To standardize and maintain integrity of the measurement protocol, all staff members who conducted the BIS measurements were trained by the first author or trainer designee personally trained by the

Table 1. Studies Evaluating Two Standard DEVIATION THRESHOLD WITH BIOIMPEDANCE SPECTROSCOPY

\begin{tabular}{|c|c|c|c|}
\hline Study & Year & $\begin{array}{l}\text { No. of } \\
\text { atients }\end{array}$ & $\begin{array}{c}\text { Findings } \\
\text { (sensitivity| specificity) }\end{array}$ \\
\hline Cornish et al. & 2001 & 162 & $\begin{array}{l}2 \text { SDs } 74 \% \mid 79 \% \text { vs. } \\
3 \text { SDs } 42 \% \mid 94 \%\end{array}$ \\
\hline Ward et al. & 2011 & 172 & $\begin{array}{l}2 \text { SDs } 68 \% \mid 89 \% \text { vs. } \\
3 \text { SDs } 37 \% \mid 100 \%\end{array}$ \\
\hline Fu et al. & 2013 & 250 & L-Dex >7.1 $80 \% \mid 90 \%$ \\
\hline Lahtinen et al. & 2015 & 100 & $\begin{array}{l}38 \text { patients with clinical BCRL; } \\
\text { BIS } 3 \text { SDs } 42 \% \mid 94 \%\end{array}$ \\
\hline Dylke et al. & 2016 & 87 & 2 SDs $76 \%-81 \% \mid 93 \%-96 \%$ \\
\hline
\end{tabular}

BCRL, breast cancer-related lymphedema; BIS, bioimpedance spectroscopy; SD, standard deviation. 
Table 2. Patient Characteristics

\begin{tabular}{|c|c|c|}
\hline Characteristic & $\mathrm{n}$ & Mean $(S D)$ \\
\hline Age & 278 & $58.6(10.6)$ \\
\hline Years of education & 277 & $14.5(2.8)$ \\
\hline Race & 280 & $\%$ \\
\hline Asian & 19 & $6.8 \%$ \\
\hline Black or African American & 22 & $7.9 \%$ \\
\hline White & 211 & $75.4 \%$ \\
\hline Other $^{\mathrm{a}}$ & 22 & $7.9 \%$ \\
\hline Do not care to respond & 6 & $2.1 \%$ \\
\hline Ethnicity & 280 & \\
\hline Non-Hispanic & 260 & $92.9 \%$ \\
\hline Hispanic or Latino & 6 & $2.1 \%$ \\
\hline Do not care to respond & 14 & $5.0 \%$ \\
\hline Marital status & 276 & \\
\hline Single & 32 & $11.6 \%$ \\
\hline Single, living with partner & 9 & $3.3 \%$ \\
\hline Married & 190 & $68.8 \%$ \\
\hline Widowed & 26 & $9.4 \%$ \\
\hline Separated & 12 & $4.3 \%$ \\
\hline Other & 7 & $2.5 \%$ \\
\hline Employment status & 277 & \\
\hline Employed full time & 119 & $43.0 \%$ \\
\hline Employed part time & 41 & $14.8 \%$ \\
\hline Homemaker & 19 & $6.9 \%$ \\
\hline Retired & 82 & $29.6 \%$ \\
\hline Unemployed & 1 & $0.4 \%$ \\
\hline On disability & 2 & $0.7 \%$ \\
\hline Other & 13 & $4.7 \%$ \\
\hline Area of residence & 276 & \\
\hline City/urban & 69 & $25.0 \%$ \\
\hline Country/rural/small town & 64 & $23.2 \%$ \\
\hline Suburb & 143 & $51.8 \%$ \\
\hline Annual household income & 276 & \\
\hline US dollars & 154 & $55.8 \%$ \\
\hline$\$ 30,000$ or less & 10 & $6.5 \%$ \\
\hline$>\$ 30,000$ & 95 & $61.7 \%$ \\
\hline Do not care to respond & 49 & $31.8 \%$ \\
\hline Australian dollars & 122 & $44.2 \%$ \\
\hline$\$ 30,000$ or less & 7 & $5.7 \%$ \\
\hline$>\$ 30,000$ & 69 & $56.6 \%$ \\
\hline Do not care to respond & 46 & $37.7 \%$ \\
\hline \multicolumn{3}{|l|}{ Insurance } \\
\hline Any government insurance & 176 & $63.1 \%$ \\
\hline Any nongovernment insurance & 211 & $75.6 \%$ \\
\hline None & 1 & $0.4 \%$ \\
\hline History of ever smoking & 87 & $31.2 \%$ \\
\hline History of ever drinking alcohol & 200 & $71.1 \%$ \\
\hline Current medications & 278 & \\
\hline Beta blockers & 22 & $7.9 \%$ \\
\hline Diuretics & 40 & $14.4 \%$ \\
\hline Oral steroids & 6 & $2.2 \%$ \\
\hline Nonsteroidal anti-inflammatories & 70 & $25.1 \%$ \\
\hline Any history of the following conditions & 279 & \\
\hline Cardiovascular & 123 & $44.1 \%$ \\
\hline Excretory & 15 & $15.4 \%$ \\
\hline Digestive & 54 & $19.4 \%$ \\
\hline Respiratory & 37 & $13.3 \%$ \\
\hline Integumentary & 54 & $19.4 \%$ \\
\hline Nervous & 32 & $11.5 \%$ \\
\hline Skeletal & 94 & $33.8 \%$ \\
\hline
\end{tabular}

TABle 2. (CONTINUED)

\begin{tabular}{lrc}
\hline Characteristic & $\mathrm{n}$ & Mean (SD) \\
\hline Muscular & 0 & $0.0 \%$ \\
Endocrine & 73 & $76.4 \%$ \\
Immune & 55 & $19.8 \%$ \\
Other & 33 & $11.9 \%$ \\
Any history of surgery & 244 & $87.5 \%$ \\
Arms & 24 & $10.0 \%$ \\
Shoulder & 10 & $4.2 \%$ \\
Trunk & 42 & $17.6 \%$ \\
\hline
\end{tabular}

${ }^{\mathrm{a}}$ Includes one multiracial, one American Indian/Alaskan Native, one Native Hawaiian/Other Pacific Islander, one Aboriginal/Torres Strait Islander.

first author. The first author made at least annual fidelity oversight visits to each research location.

Baseline assessments were conducted before any cancer treatment. At 2 months postsurgery, participants were censored if they did not have at least one of the following: mastectomy, axillary dissection (ALND), $>6$ nodes removed during a sentinel node procedure, radiation therapy, or taxane chemotherapy. The remaining participants were randomized within site to either BIS or TM and to be monitored at 3-6month intervals up to 36 months postsurgery. An L-Dex U400 (ImpediMed Limited, Brisbane, Australia) was utilized. For BIS measurements, participants were placed in the supine position, and skin preparation and electrode placement were per the manufacturer's instructions. ${ }^{12}$ Based on

Table 3. Clinical Characteristics at 2 Months PostFinal Surgery

\begin{tabular}{lrr}
\hline Characteristic & $\mathrm{n}$ & $\%$ \\
\hline Breast cancer stage & 280 & \\
DCIS & 10 & 3.6 \\
I & 150 & 53.6 \\
II & 93 & 33.2 \\
III & 27 & 9.6 \\
Surgery & 280 & \\
Mastectomy & 56 & 20.0 \\
Breast conservation & 224 & 80.0 \\
Axillary management & 280 & \\
Yes (meeting trial entry criteria) & 74 & 26.4 \\
Any axillary dissection & 64 & 86.5 \\
Any sentinel node biopsy (>6 nodes) & 24 & 32.4 \\
No & 206 & 73.6 \\
Radiation therapy & 280 & \\
Yes & 186 & 66.4 \\
Chest wall/breast & 181 & 97.8 \\
Axilla & 33 & 18.1 \\
Supraclavicular fossa & 10 & 5.5 \\
No & 94 & 33.6 \\
Taxane-based chemotherapy & 280 & \\
Yes & 106 & 37.9 \\
Planned & 23 & 21.7 \\
Completed & 27 & 25.5 \\
Ongoing & 56 & 52.8 \\
No & 174 & 62.1 \\
\hline
\end{tabular}

(continued) DCIS, ductal carcinoma in situ. 
evidence at the time of the initiation of the parent study, the initial cohort prevention intervention trigger threshold for the L-Dex group was a change from baseline of $\geq 10$ L-Dex units. ${ }^{13-14}$ Participants meeting this prespecified trigger were treated with a compression sleeve and gauntlet for 28 days at a dose of 12 hours per day.

\section{Statistical analysis}

Frequency distributions were used to summarize the nominal and ordinal patient characteristics. Mean and SD summarized normally distributed continuous data; median and interquartile range (IQR) summarized skewed distributions. Bootstrapped 95\% CIs were generated around the observed L-Dex baseline and change from baseline values.

\section{Results}

A total of 280 women with a baseline and at least one L-Dex measurement within the 12-month postsurgery timeframe period were included in this analysis. Median follow-up for this sample was 9 months postsurgery (IQR:
6-12 months) with a median of three measurements per patient (IQR: 3-4). Patient, tumor, and treatment characteristics are presented in Tables 2 and 3. Mean age for the cohort was 58.6 years with $20 \%(n=56)$ undergoing mastectomy. With respect to axillary management, $26 \%(n=74)$ had axillary surgery meeting inclusion criteria, with $86.5 \%$ of those participants $(n=64)$ undergoing ALND. Overall, $66.4 \%$ of participants $(n=186)$ received radiation therapy with $23.6 \%(n=43)$ receiving some form of regional nodal irradiation. In addition, $37.9 \%(n=106)$ received taxane chemotherapy.

\section{Pretreatment L-Dex}

Pretreatment L-Dex values are presented in Figure 1. The distribution was slightly positively skewed with a mean value of 0.007 and median value of 0.200 , with the median felt to be more representative due to the skew. The bias-corrected bootstrapped $95 \%$ CI for the median pretreatment L-Dex value was -0.500 to +0.800 . The documented normative mean value is 0.00 , which is at the 49 th percentile for this sample. Approximately 6\% $(n=18)$ had a baseline L-Dex

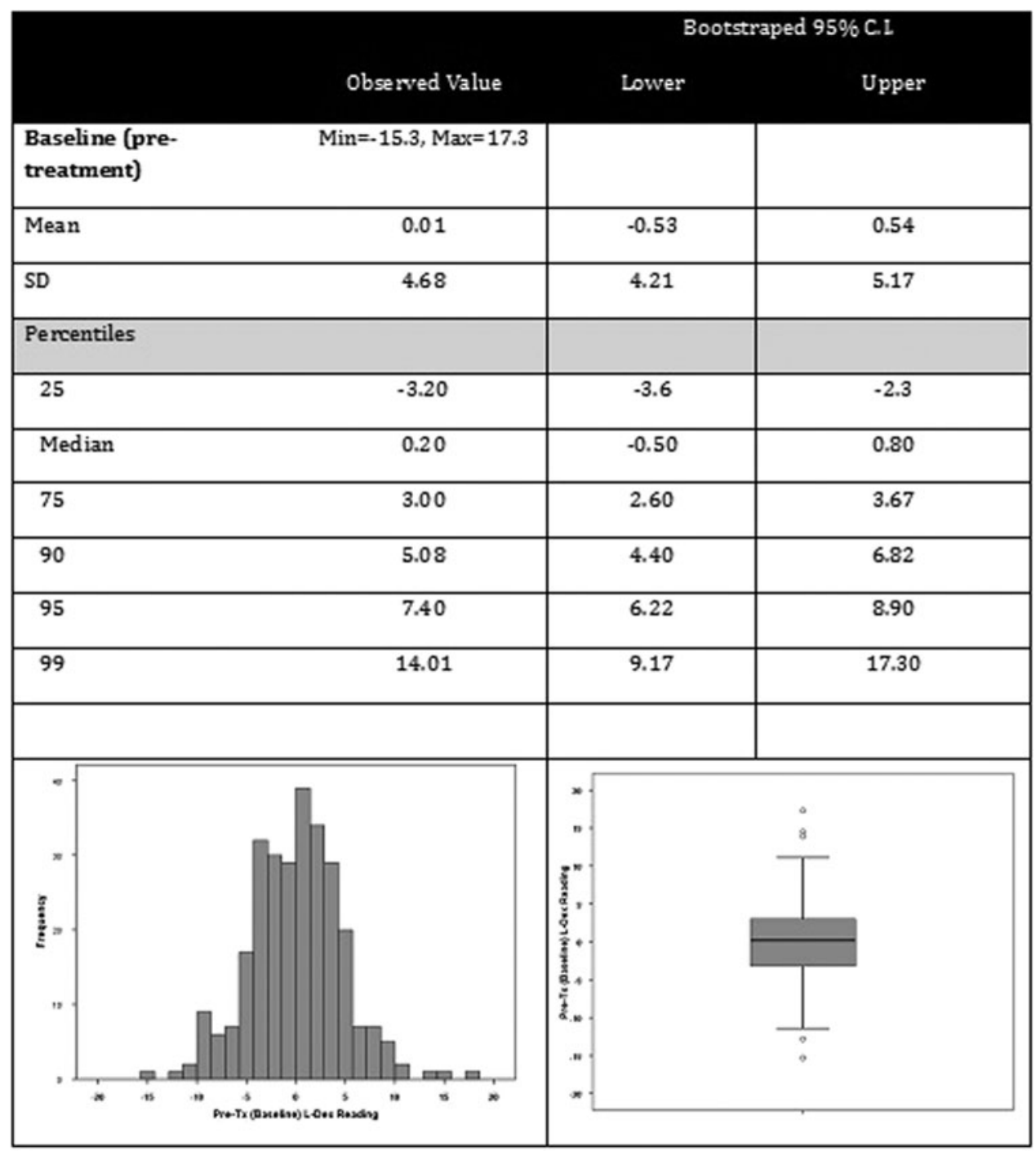

FIG. 1. Baseline (pretreatment) assessments. 
value of $\geq 7.0$ and $1.8 \%$ of participants $(n=5)$ had an L-Dex value $\geq 10.0$.

\section{Maximum 1-year change L-Dex}

Summaries of the maximum change in L-Dex values within the first year after surgery are presented in Figure 2. Maximum change was noted at a median of 6.0 months postsurgery. The IQR for maximum change from pretreatment was 4-9 months postsurgery, indicating that $50 \%$ of the participants reached maximum change within that time period, yet $25 \%$ had maximal change before 4 months and $25 \%$ between the 9 and 12 months postsurgery time interval studied. The median maximum L-Dex change was 2.10 with a bias-corrected $95 \%$ CI of +1.55 to +3.00 . Based on the observed changes in this sample, $<10 \%(7.5 \%)$ of participants diagnosed and treated for breast cancer would be expected to have a volume change of $10 \%$ from baseline or an L-Dex unit change of 10 (current criteria based on normative values). Twenty percent of these women would be expected to have a change $\geq 6.5$ L-Dex units $(95 \%$ $\mathrm{CI}=5.50-7.30)$ and $15 \%$ a change of $\geq 7.4 \mathrm{~L}$-Dex units $(95 \%$ $\mathrm{CI}=6.50-8.40)$. In our sample, $17.1 \%(n=48)$ had a change in L-Dex value from baseline of $\geq 7.0 \mathrm{~L}-$ Dex units.

\section{Conclusions}

This study provides information regarding absolute L-Dex values present in women newly diagnosed with breast cancer. Findings from the baseline pretreatment data in this study demonstrate a variability that is only slightly larger (4 units as compared to 3 units) than the previously documented absolute normative L-Dex values, yet due to skewness, only $6 \%$ of women within this population can be expected to have an absolute L-Dex value of $\geq 7.0$. Thus, based on absolute L-Dex values, our findings do not support pre-existing lymphedema as a common occurrence in women newly diagnosed with breast cancer.

In addition to better defining pretreatment L-Dex readings among women with breast cancer, readings from this large cohort were obtained up to 12 months postsurgery. This represents the largest study known to date that has longitudinally examined L-Dex measurements over this period. Change in L-Dex readings from a known pretreatment L-Dex baseline rather than an absolute L-Dex value is currently used in both research and clinical settings to trigger further assessment and possible intervention for those with breast cancer, but prospective data from such a large cohort have been limited. During the 12 month follow-up period, $17.1 \%$

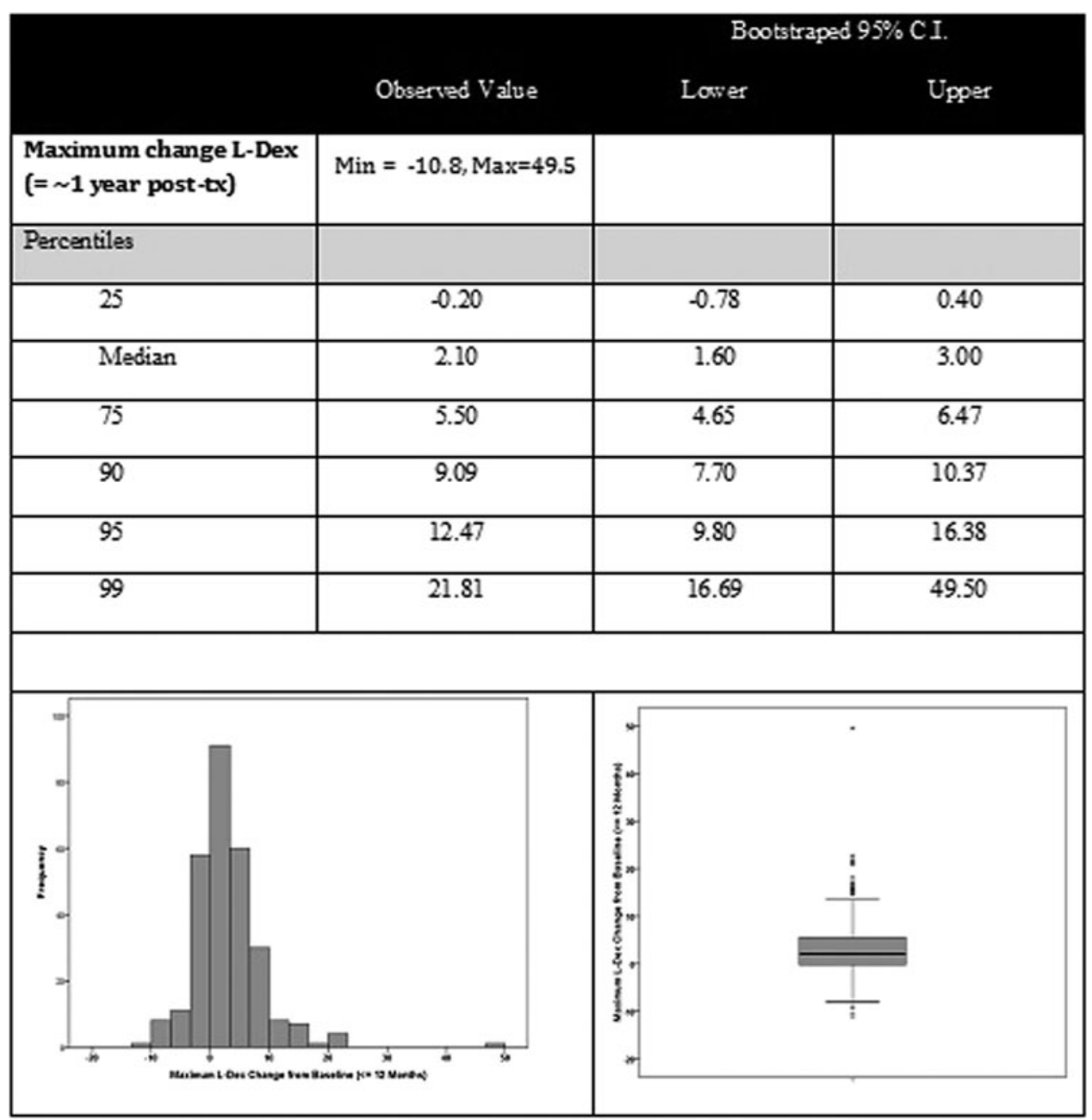

FIG. 2. Maximum change L-Dex ( $\leq \sim 1$ year postsurgery). 
of the 280 participants in this study had a maximum L-Dex change of $\geq 7.0 \mathrm{~L}$-Dex units from their respective individual baseline value. This is consistent with recent changes in lymphedema diagnostic criteria using L-Dex, and in agreement with recent publications, and suggests a significant percentage of individuals may develop clinical or subclinical lymphedema within a few short months of completing surgery. ${ }^{4,-11}$ Historically, rates of lymphedema have varied significantly with different measurement methods, timing of measurement, and criteria for diagnosis, so direct comparisons with previous studies are difficult. ${ }^{11,13}$ The $17.1 \%$ rate of clinical lymphedema identified in this study is higher than the $11.4 \%$ rate found in a previous study utilizing BIS; however, the previous study that found a rate of $11.4 \%$ used a higher absolute value of 3 SDs above normative as the criteria for diagnosis and assessed the first 6 months postsurgery and, therefore, a higher rate of BCRL would be expected with the use of a more sensitive criteria and a longer period of follow-up. ${ }^{17}$ The BCRL rate in this study is also slightly higher than the rate of $16 \%$ reported 1 -year postsurgery as determined by a maximum increase in arm circumference as measured by TM, which may be attributed to the higher sensitivity of BIS than TM. ${ }^{15}$ However, the current results are well within the $7 \%-70 \%$ rates of lymphedema within the first 6 months of treatment noted in a review article and within the assumed rate (based upon eligibility criteria for study enrollment) used to power the parent study of $20 \%$. $^{15}$

The maximum change in L-Dex readings ranged from -10.08 through 49.95 . Some baseline values were high; therefore, a decrease in these values over time was anticipated in some participants. The initial high L-Dex values in a subset of the participants do raise questions as to the biological mechanisms underlying these elevated baseline readings. Another important finding from this analysis is that 6 months postsurgery was the median time to when maximum L-Dex change occurred. Given consistent data that demonstrate that early detection of lymphedema improves patient outcomes, aggressive measurement with L-Dex at a frequency greater than every 6 months, that is, every 3 months, especially during the first 6-12 months postsurgery, is recommended to facilitate identification of subclinical lymphedema and support early diagnosis of clinical lymphedema. $5,12,16,17$

Recent publications have established a lower L-Dex threshold for clinical lymphedema, and are consistent with 2 SDs above the mean rather than 3 SDs, to increase the sensitivity to detect clinical lymphedema (Table 1). Therefore, based upon careful evaluation of our findings, Stout et al.'s previous work, protection of human subject considerations, and the recent revised diagnostic threshold of $\geq 7.0 \mathrm{~L}-\mathrm{Dex}$ units for clinical lymphedema, ${ }^{4,8-11}$ we recommend that an L-Dex change from baseline approximating $\geq 6.5$, rather than $\geq 10$, be considered to best capture subclinical lymphedema in those with a history of breast cancer. Although future studies may more precisely define the subclinical threshold, use of this recommended lower threshold may enhance the potential success of prevention interventions in the breast cancer population at risk for developing lymphedema.

Findings in this study should be considered in light of its limitations. The median follow-up for this cohort of participants is short; however, this should have no impact on initial L-Dex values or maximum change in L-Dex values during the first year. In addition, although there were few events in this cohort, because of the fact that the analysis looked at maximum change, this is not likely to influence the results presented. Finally, the study from which these participants were evaluated continues to accrue with completion expected in early 2018 with results to be published thereafter. In the meantime, these results support the integration of LDex measurements in the clinical surveillance of breast cancer survivors at risk for developing clinical lymphedema. Our findings do not contradict the recent body of work supporting a change to the use of an absolute L-Dex value of $\geq 7$ (representative of $\sim 2$ SDs from normative mean values of " 0 ") as indicative of lymphedema. ${ }^{4-11}$ In light of the present data and these studies, IRB and SRC approvals were obtained to modify the prevention intervention trigger in the parent study from $\geq 10$ L-Dex unit change to $\geq 6.5$ L-Dex units.

\section{Author Disclosure Statement}

Chirag Shah is scientific consultant for ImpediMed Limited. Frank Vicini is scientific consultant and chief medical officer for ImpediMed Limited.

Funded by grant support for REDCap ${ }^{\mathrm{TM}}$ (UL 1TR000445) from NCATS/NIH. Impedimed and medi.

\section{References}

1. Ward LC, Bunce IH, Cornish BH, et al. Multi-frequency bioelectrical impedance augments the diagnosis and management of lymphoedema in post-mastectomy patients. Eur J Clin Invest 1992; 22:751-754.

2. Lawenda BD, Mondry TE, Johnstone PA. Lymphedema: A primer on the identification and management of a chronic condition in oncologic treatment. CA Cancer J Clin 2009; 59:8-24.

3. Whitley E, Ball J. Statistics review 2: Samples and populations. Crit Care 2002; 6:143-148.

4. Cornish BH, Chapman M, Hirst C, et al. Early diagnosis of lymphedema using multiple frequency bioimpedance. Lymphology 2001; 34:2-11.

5. Stout Gergich NL, Pfalzer LA, McGarvey C, et al. Preoperative assessment enables the early diagnosis and successful treatment of lymphedema. Cancer 2008; 112:2809-2819.

6. National Lymphedema Network. NLN Position Paper: The Diagnosis and Treatment of Lymphedema. Available at: https://www.lymphnet.org/resources/nln-position-paper-thediagnosis-and-treatment-of-lymphedema (Last accessed August 15, 2017).

7. Ward L. Is BIS ready for prime time as the gold standard measure? J Lymphoedema 2009; 4:52-56.

8. Ward LC, Dylke E, Czerniec S, et al. Confirmation of the reference impedance ratios used for assessment of breast cancer-related lymphedema by bioelectrical impedance spectroscopy. Lymphat Res Biol 2011; 9:47-51.

9. Lahtinen T, Seppala J, Viren T, et al. Experimental and analytical comparison of tissue dielectric constant (TDC) and bioimpedance spectroscopy (BIS) in assessment of early arm lymphedema in breast cancer patients after axillary surgery and radiotherapy. Lymphat Res Biol 2015; 13:176-185.

10. Dylke ES, Schembri GP, Bailey DL, et al. Diagnosis of upper limb lymphedema: Development of an evidencebased approach. Act Oncologica 2016; 55:1477-1483. 
11. Fu M, Cleland C, Guth A, et al. L-Dex ${ }^{\circledR}$ ratio in detecting breast cancer-related lymphedema: Reliability, sensitivity and specificity. Lymphology 2013; 46:85-96.

12. Shah C, Vicini FA, Arthur D. Bioimpedance spectroscopy for breast cancer related lymphedema assessment: Clinical practice guidelines. Breast J 2016; 22:645-650.

13. Armer J, Stewart BR. A comparison of four diagnostic criteria for lymphedema in post-breast cancer population. Lymphat Res Biol 2005; 3:208-217.

14. Hayes S, Janda M, Cornish B, et al. Lymphedema after breast cancer: Incidence, risk factors, and effect on upper body function. J Clin Oncol 2008; 26:3536-3542.

15. Hayes S, Cornish B, Newman B. Comparison of methods to diagnose lymphedema among breast cancer survivors: 6month follow-up. Breast Cancer Res Treat 2005; 89:221226.

16. Torres Lacomba M, Yuste Sancez MJ, Zapico Goni A, et al. Effectiveness of early physiotherapy to prevent lymphedema after surgery for breast cancer: Randomised, single blinded, clinical trial. BMJ 20910; 340:b5396.

17. Soran A, Ozmen T, McGuire KP, et al. The importance of detection of subclinical lymphedema for the prevention of breast cancer-related clinical lymphedema after axillary lymph node dissection; a prospective observational study. Lymphat Res Biol 2014; 12:289-294.

Address correspondence to: Sheila H. Ridner, PhD, RN, FAAN Vanderbilt University School of Nursing 525 Godchaux Hall 461, 21st Avenue, South Nashville, TN 37240

E-mail: Sheila.ridner@vanderbilt.edu 